\title{
Myeloid Sarcoma in the Nasal Cavities that Developed during the Course of Acute Myelomonocytic Leukemia
}

\author{
Yasuhiro Yamamoto, ${ }^{1)}$ Tsukasa Uchima, ${ }^{2)}$ Yoshizumi Konoike, ${ }^{1)}$ and Hirokazu Nakamine ${ }^{3)}$
}

Keywords: acute myelomonocytic leukemia, myeloid sarcoma, nasal tumor

\section{To the Editor}

In December 2006, a 95-year-old Japanese woman was transferred to our hospital for the evaluation of her leukocytosis. In September 2006, the patient was medicated for hypertension by her family physician and she was found to have leukocytopenia $(2,500 / \mu \mathrm{L})$ without abnormal cells in the peripheral blood (PB). During the middle of November, the patient's white blood cell (WBC) count was found to be elevated. Upon admission to our hospital, the patient had no complaints and presented no remarkable findings on physical examination. She had no clinical evidence of dementia. A complete blood count showed WBC count of $58,300 / \mu \mathrm{L}$ (myeloblasts $7 \%$, neutrophils $27 \%$, metamyelocytes $1 \%$, monocytes $58 \%$, and lymphocytes $7 \%$ ), hemoglobin of 9.1 $\mathrm{g} / \mathrm{dL}$, and platelet count of $149,000 / \mu \mathrm{L}$. Most of the monocytes were immature and positive for CD11b, CD13, CD14, CD15, CD33, and CD64. Hypersegmented neutrophils and giant neutrophils were seen. Since the patient and her family refused bone marrow aspiration (BMA), we considered from the above PB profile and the progressive clinical features that the patient likely had acute myelomonocytic leukemia (AMMoL). Following chemotherapy with low-dose cytarabine (Ara-C) (20 mg/day subcutaneously, on days 1-25), her WBC count gradually decreased, and myeloblasts and abnormal monocytes disappeared. Her general condition was seen to be stable.

At the end of March 2007, her WBC count again elevated with myeloblasts and abnormal monocytes $(15 \%$ and $8 \%$,

\section{Received : July 18, 2009}

Revised : December 27, 2009

Accepted : January 7, 2010

1)Department of Internal Medicine, Heisei Memorial Hospital, Nara, Japan

${ }^{2}$ Department of Laboratory Medicine, Heisei Memorial Hospital, Nara, Japan

${ }^{33}$ Division of Pathology and Immunology, Kansai University for Medical Science, Osaka, Japan

Address correspondence and reprint request to Yasuhiro Yamamoto, M.D., Department of Internal Medicine, Heisei Memorial Hospital, Shijyo-cho 827, Kashihara, Nara 634-0813, Japan

E-mail : yyama@mx5.canvas.ne.jp respectively) being around $10,000 / \mu \mathrm{L}$. BMA could be carried out at that time, and it showed elevation of myeloblast and monoblast percentages (19.3\% and 22.5\%, respectively) (Fig. 1). Blasts were positive for myeloperoxidase (MPO) and monoblasts were positive for non-specific esterase, CD11b, and CD64. Since dysplasia in the myeloid series, characterized by hypersegmented neutrophils, giant neutrophils and Pelger-Hüet nuclear anomaly, was detected, a diagnosis of AMMoL, which had been most likely progressed from myelodysplastic syndrome (MDS), was further confirmed. The possibility that the present case was chronic myelomonocytic leukemia could be excluded because of the presence of excessive myeloblasts. Chromosomal analysis of BM revealed no abnormalities. At that time, the patient also presented bilateral nasal obstruction with swelling. A computed tomography (CT) scan revealed a soft tissue mass occupying both of the nasal cavities by involving the nasal septum (Fig. 2a, b). No destruction of the bone was evident. Biopsy of the tumor showed diffuse proliferation of large mononuclear cells which was covered by focally erosive mucosal tissue with the infiltration of mature neutrophils and plasma cells. The large mononuclear cells had immature-appearing nuclei with mitotic features and weakly eosinophilic cytoplasm. (Fig. 3a, b). Immunohistochemically, the cells were positive for MPO, $a_{1^{-}}$ antitrypsin (AAT), lysozyme, CD4, CD15, CD68, and $\mathrm{CD} 163$, and were negative for $\mathrm{CD} 3 \varepsilon, \mathrm{CD} 20, \mathrm{CD} 34, \mathrm{CD} 56$, and CD123 (Fig. 4). Among the proliferating cells, MPO, AAT, lysozyme, and CD68 were positive in the vast majority of cells, while the positive rates of CD15, CD4, and CD163 were estimated to be $80 \%, 60 \%$, and $40 \%$, respectively. Although these findings are not precise, the proliferating cells appeared to be composed of granulocytic, monocytic, and granulomonocytic (a cell having both granulocyte-related and monocyte-related molecules) lineages. Based on these findings, the lesion was diagnosed as one of myeloid sarcoma (MS), and could be classified as myelomonocytic sarcoma. Chemotherapy consisting of low-dose Ara-C (20 mg/day subcutaneously, on days 1-14) and aclarubicin (10 mg/day intravenously, on days 1-4) resulted in a decrease of WBC count 


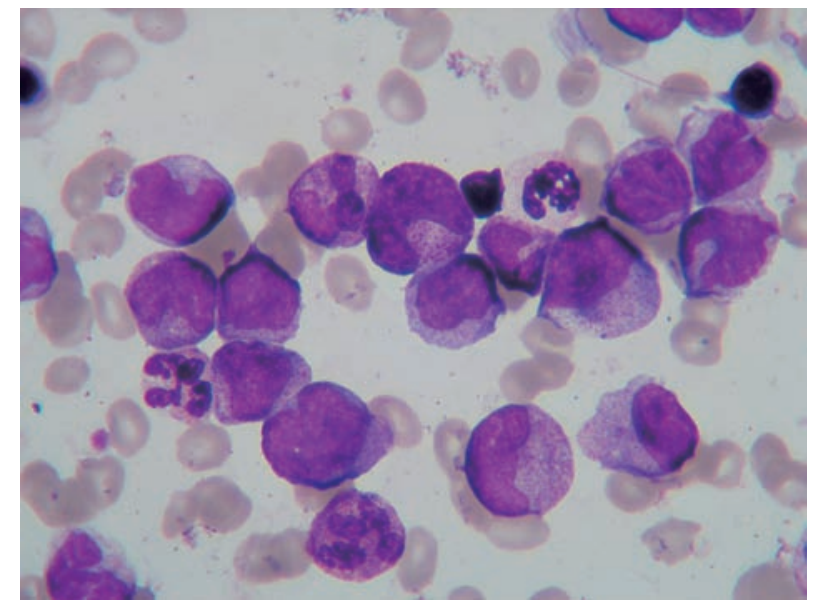

Fig. 1. Bone marrow aspirate smear shows increase of myeloblasts and monoblasts. Wright-Giemsa stain, $\times 100$.
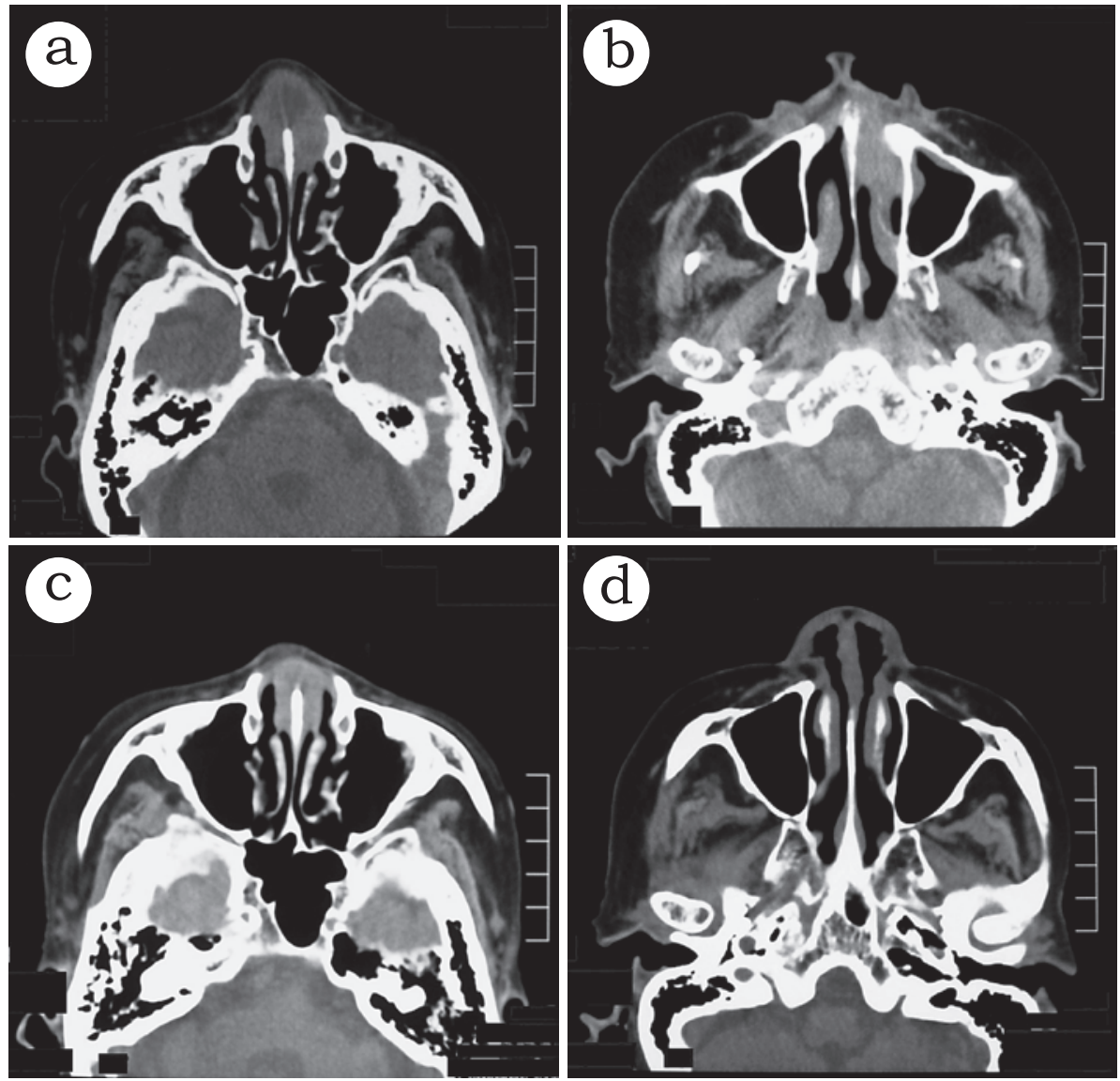

Fig. 2. Computed tomography scan of the nasal tumor. $(\mathbf{2} \boldsymbol{a}, \boldsymbol{b})$ The tumor spread to both of the nasal cavities by involving the septum before chemotherapy. (2c, d) The tumor decreased in size after chemotherapy. 

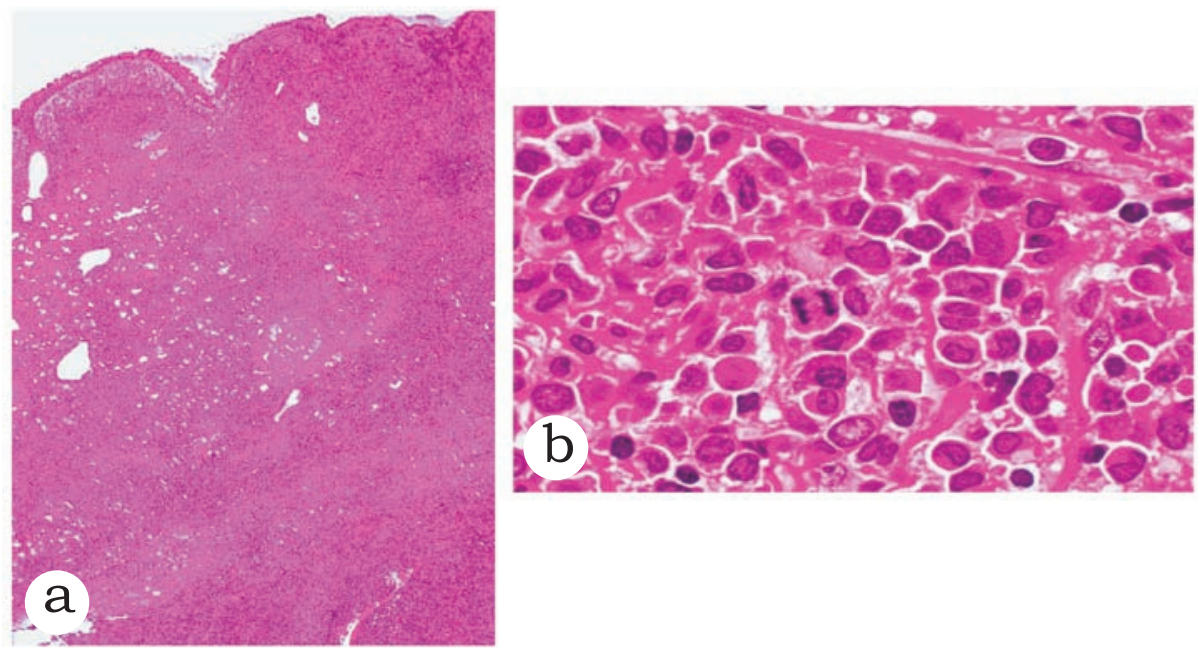

Fig. 3. Histologic features of the biopsy sample. (3a) The upper respiratory tract shows erosion with the infiltration of mature-appearing neutrophils and plasma cells. $(3 \boldsymbol{b})$ Beneath these cells, diffuse proliferation of large mononuclear cells is seen. H\&E stain, $(3 \boldsymbol{a}) \times 4,(3 \boldsymbol{b})$ $\times 100$.
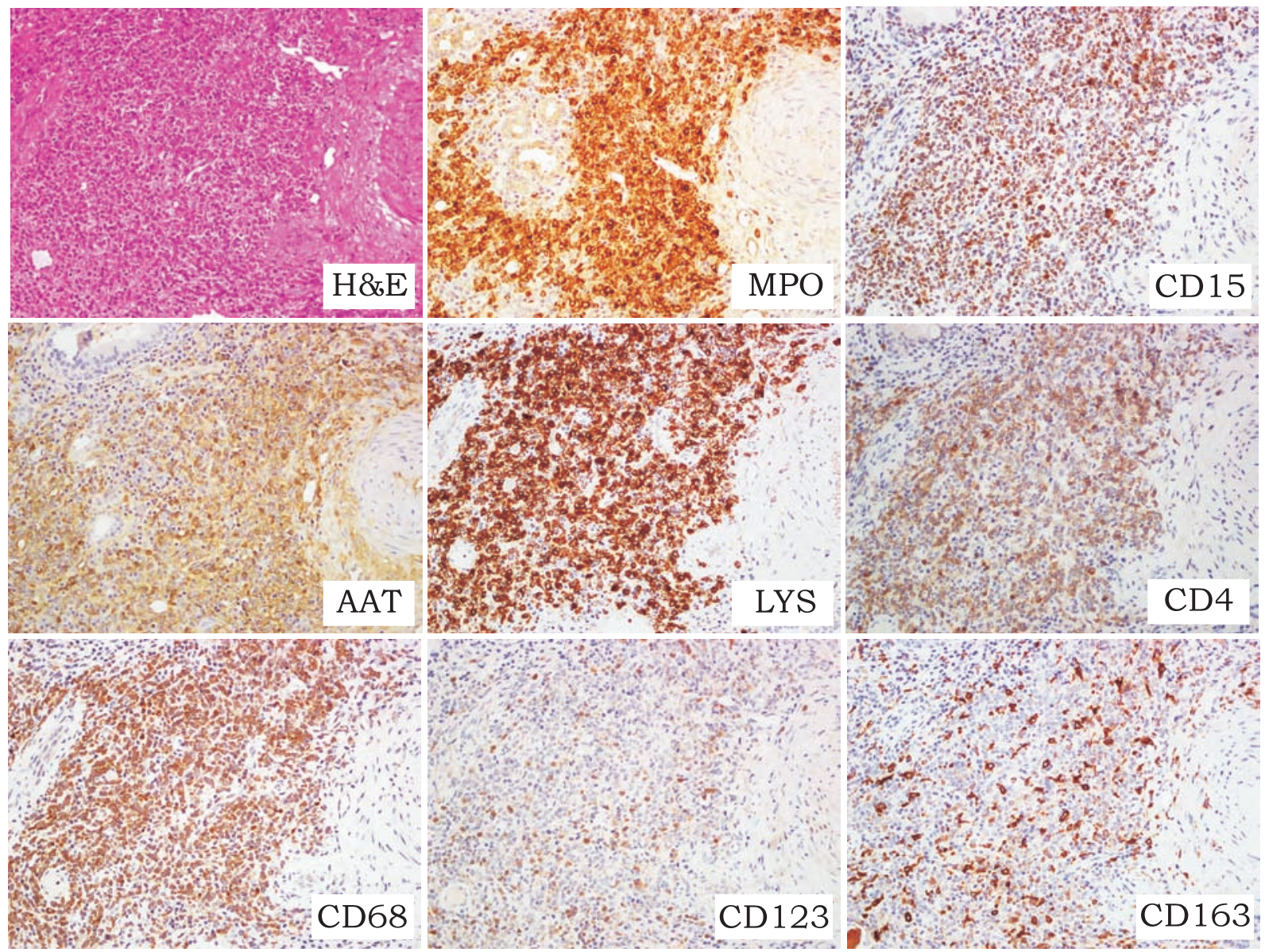

Fig. 4. H\&E stain and immunohistochemical stain of the biopsy sample. The large cells are positive for myeloperoxidase, $a_{1}$-antitrypsin, lysozyme, CD4, CD15, CD68, and CD163, and are negative for CD123. Counterstained with hematoxylin in cases of immunostain, All figures, $\times 20$. 
and disappearance of blasts in PB. The nasal tumor also decreased in size (Fig. 2c, d) and the patient's symptoms were relieved. Although WBC and blast counts elevated again in July and once again in September, low-dose Ara-C was effective in both instances and her relatively good general condition was maintained. At the end of September 2007, however, the patient developed a case of sudden cardiac arrest and expired. On that day, the percentage of blasts in PB was as low as $2 \%$ (day 9 of low-dose Ara-C). The direct cause of her death was inconclusive and there was no recurrence of nasal tumor.

MS is defined as a tumor mass of myeloid blasts with/ without maturation occurring at an anatomical site other than bone marrow with normal architectural effacement. ${ }^{1}$ It is especially important to identify destructive growth to characterize the lesion to be MS in leukemic patients. In our case, the tumor occupied both of the nasal cavities via the nasal septum and it showed irregular border to the surrounding tissue on CT scan. Thus, the lesion was diagnosed as one of "secondary" MS, but not simple tissue infiltration of leukemic cells, although bone destruction was not evident.

To our knowledge, there have been only a few reports of MS occurring in the nasal cavity, irrespective of whether the lesion was either de novo or secondary to leukemia., ${ }^{2,3} \mathrm{MS}$ is often misdiagnosed as non-Hodgkin's lymphoma (NHL), ${ }^{4,5}$ especially in case of de novo lesions, in the absence of proper immunohistochemical studies. Pileri et al. reported 92 cases of MS, 25 cases $(27 \%)$ of which were considered to be de novo, and 10 of the latter 25 cases were misdiagnosed as NHL. ${ }^{5}$ Reports regarding the prognosis of patients with MS are controversial. The above authors emphasized that autologous or allogeneic bone-marrow transplantation was essential to achieve complete remission and cure for both de novo MS and MS related to acute myeloid leukemia (AML), MDS, or myeloproliferative disorders. ${ }^{5}$ On the other hand,
Tsimberidou et al. recently reported that de novo MS was associated with a superior prognosis compared with AML. ${ }^{6}$ In this extremely elderly patient, the local control of the nasal tumor through chemotherapy was considered successful.

Diagnosis of MS developing in the course of AML, as in our case, is not difficult. However, MS should be added to differential diagnoses for "undifferentiated" nasal tumors, especially when a given patient has MDS.

\section{REFERENCES}

1 Pileri SA, Orazi A, Falini B : Myeloid sarcoma. In : Swerdlow SH, Campo E, Harris NL, Jaffe ES, Pileri SA, et al. (eds) : World Health Organization Classification of Tumours, WHO Classification of Tumours of Haematopoietic and Lymphoid Tissues. 4th ed, International Agency for Research on Cancer (IARC), Lyon, pp. 140-141, 2008

2 Prades JM, Alaani A, Mosnier JF, Dumollard JM, Martin C: Granulocytic sarcoma of the nasal cavity. Rhinology 40: 159161,2002

3 Teramoto H, Miwa H, Patel V, Letwin N, Castellone MD, et al. : Gene expression changes in a patient presenting nonleukaemic nasal granulocytic sarcoma to acute myelogenous leukaemia using 40 K cDNA microarray. Clin Lab Haematol 28 : 262-266, 2006

4 Paydas S, Zorludemir S, Ergin M: Granulocytic sarcoma : 32 cases and review of the literature. Leuk Lymphoma $47: 2527-$ 2541, 2006

5 Pileri SA, Ascani S, Cox MC, Campidelli C, Bacci F, et al.: Myeloid sarcoma : clinico-pathologic, phenotypic and cytogenetic analysis of 92 adult patients. Leukemia 21 : 340-350, 2007

6 Tsimberidou AM, Kantarjian HM, Wen S, Keating MJ, O’Brien S, et al.: Myeloid sarcoma is associated with superior event-free survival and overall survival compared with acute myeloid leukemia. Cancer. 113 : 1370-1378, 2008 